\title{
A IMPORTÂNCIA DO FEEDBACK E DAS CRENÇAS DE AUTOEFICÁCIA DO ALUNO NA AQUISIÇÃO DE LÍNGUA ESTRANGEIRA
}

\author{
Carmem Silvia Lima Fluminhan ${ }^{1}$, Camélia Santina Murgo ${ }^{1}$, Antonio Fluminhan $^{2}$ \\ Universidade do Oeste Paulista - UNOESTE, ${ }^{1}$ Mestrado em Educação, ${ }^{2}$ Mestrado em Meio Ambiente e \\ Desenvolvimento Regional, Presidente Prudente, SP. Email: carmem.slima@hotmail.com
}

\begin{abstract}
RESUMO
O uso do feedback como ferramenta pedagógica na aquisição de língua estrangeira tem sido pesquisado com crescente interesse nos últimos anos. Muito se tem escrito sobre o emprego de feedback em ambientes educacionais, e as razões pelas quais o feedback é um instrumento eficaz na promoção da autonomia e do desenvolvimento linguístico do aluno. Este artigo pretende colaborar para esclarecer de que forma o uso adequado do feedback por parte do professor e as crenças de autoeficácia do aluno são fundamentais para o desenvolvimento da aprendizagem de língua estrangeira. Entretanto, nota-se que existe uma grande necessidade de estabelecer a relação entre o recebimento adequado de feedback e as crenças de autoeficácia do aluno. Esta é uma lacuna significativa, pois, tanto o ponto de vista do professor quanto as crenças acima mencionadas por parte do aluno influenciarão de forma decisiva o uso eficaz deste instrumento pedagógico.
\end{abstract}

Palavras-chave: Feedback, autoeficácia, aprendizagem, autonomia pessoal, língua estrangeira.

\section{THE IMPORTANCE OF FEEDBACK AND THE STUDENT'S SELF-EFFICACY BELIEFS IN THE FOREIGN LANGUAGE AQUISITION}

\begin{abstract}
The use of feedback as a pedagogical tool in the acquisition of a foreign language has been studied with growing interest in recent years. Much has been written about the use of feedback in educational environments, as well as the reasons why feedback is an effective tool in promoting student's autonomy and linguistic development. This article seeks to contribute to clarify how the proper use of the teacher's feedback and the student's self-efficacy beliefs are crucial to the development of a foreign language acquisition. However, there is still a major necessity to establish the relation between receiving proper and consistent feedback and student's selfefficacy beliefs. This is a significant gap, since both the teacher's point of view and the student's self-efficacy beliefs will decisively influence the effective use of this pedagogical tool.

Keywords: Feedback, self-efficacy, learning, personal autonomy, foreign language
\end{abstract}




\section{INTRODUÇÃO}

No modelo de ensino de idiomas presencial, em que o professor está em contato direto com os alunos, a utilização do feedback é de suma importância para inúmeras situações, tais como: responder dúvidas, avaliar e acompanhar o aprendiz no desenvolvimento das quatro habilidades do idioma (ouvir, falar, ler e escrever) de forma motivadora, que impulsione o aluno a alcançar os seus objetivos, entre outras.

O feedback no ambiente educacional tem sido alvo de estudos de vários pesquisadores (ANDERSON et al., 1995; MERRIL et al., 1992; HUME et al., 1996; CHI et al., 2001, FLORES, 2009; CARDOSO, 2011). Entretanto, pouca atenção tem sido dada ao feedback como ferramenta para a aprendizagem específica de língua estrangeira (LE). Tal situação pode ser explicada pelas seguintes razões, entre elas: a) a natureza específica e complexa dos erros e o feedback corretivo na aprendizagem de LE; b) a eficácia do uso dos diferentes tipos de feedback no processo de ensino e de aprendizagem de LE ainda não tem sido compreendida; c) a falta de pesquisa empírica sobre a eficácia do feedback no processo de aquisição de LE e estratégias específicas para o seu uso em sala de aula durante a aquisição de LE; d) a falta de conhecimento dos resultados das pesquisas realizadas pelos pesquisadores de feedback no processo de ensino e de aprendizagem de LE.

O erro e o feedback corretivo ocorrem naturalmente durante o processo de ensino e de aprendizagem de LE em sala de aula. Os erros podem ser definidos como desvios da norma culta na língua alvo (ELLIS, 1997). O feedback corretivo é uma indicação ao aprendiz de que a língua alvo foi usada incorretamente. Segundo Ellis, 1997, o tratamento que é dado ao erro é um processo amplo e complexo. Cabe ao professor, portanto, identificar e definir quais erros e em que momento a correção deve acontecer. Além disso, há diferentes tipos de feedback descritos na literatura especializada, bem como diversas estratégias foram propostas. No entanto, pouco tem se discutido como utilizar o feedback em sala de aula em curso de idioma como LE.

Pesquisas realizadas acerca do uso de feedback no processo de aquisição de LE revelam que os professores possuem uma variedade ampla de estratégias para identificar e tratar o erro (ALLWRIGHT, 1975 e LYSTER e RANTA, 1997). Contudo, os estudos sistemáticos que investigaram o tipo, a frequência e a eficácia do feedback são mais recentes (GUS, 2001; PAIVA, 2003; FERREIRA， 2007,2011; FLORES， 2009, CARDOSO, 2011). Os resultados de tais investigações apontam que (1) o benefício do uso dos diferentes tipos de feedback ainda 
não está claro e (2) a eficácia das estratégias de feedback depende de múltiplos fatores, incluindo aspectos específicos que se pretende corrigir, o tempo disponível para a correção, e características dos alunos (por exemplo, explicações gramaticais profundas não são adequadas para alunos iniciantes).

Durante a correção do erro em ambiente de aprendizagem de LE, algumas estratégias de feedback têm sido comumente usadas pelos professores, tais como, o feedback meta-linguístico (quando o professor comenta, informa ou questiona o aluno acerca do erro cometido, como por exemplo, "Don't forget the verb agrees with the subject") e a identificação do erro (quando o professor informa ao aluno que houve um erro e aponta o local na frase onde o erro foi cometido) (CRISWELL et al., 1991; SAMS, 1995; NAGATA, 1995). Entretanto, tais estratégias se mostram obstrutivas e artificiais durante as atividades de conversação ou de comunicação em sala de aula, sendo apenas eficazes para a correção de exercícios gramaticais, onde o foco está na forma linguística.

Assim sendo, o professor de LE ainda não dispõe de recursos que the forneça embasamento teórico para incorporar em sua rotina de trabalho estratégias específicas de feedback. Muitos profissionais da área de educação de LE não têm conhecimento de que o feedback é objeto de pesquisa científica e que, recentemente, o interesse pelo assunto tem aumentado no meio acadêmico, como provam os números crescentes de publicações sobre o assunto.

Apesar dos problemas citados, o valor do feedback é inquestionável no processo de ensino e de aprendizagem de LE (GARRETT, 1995). Entretanto, é necessário determinar até que ponto $\mathrm{o}$ feedback realmente contribui para que o aluno desenvolva a língua alvo, e como consequência, é fundamental que obtenhamos mais detalhes de como os aprendizes respondem às estratégias de feedback, e se tais estratégias resultam em mudança no processo de aprendizagem.

A presente pesquisa se propõe a verificar como o feedback pode ser usado como uma ferramenta eficaz na aprendizagem de língua estrangeira. Além disso, a pesquisa se preocupa em relacionar de que forma a crença de autoeficácia do aluno pode influenciá-lo em aceitar o próprio erro, corrigi-lo satisfatoriamente e, como consequência, alcançar o desempenho desejado como maior facilidade.

\section{A FERRAMENTA FEEDBACK}

A presente pesquisa foi realizada através de um extenso levantamento de obras sobre o tema, tais como: Cohen e Cavalcanti (1990), Keh (1990), Scherman (1994), Kulhavy (1997), Gus (2001), Shute 
(2007), Cardoso (2007; 2011), Flores, (2009), entre outros, que argumentam que o uso do feedback produz resultados amplamente positivos, tais como: a melhora na qualidade do aprendizado, maior participação dos alunos nas tomadas de decisão em relação ao seu aprendizado, o indivíduo torna-se melhor preparado para a autocorreção e, portanto, desenvolve maior independência nos estudos e, finalmente, permite que o aluno use melhor as oportunidades de aprendizagem dentro e fora da sala de aula.

Abordagens sobre o emprego do feedback avaliativo foram realizadas por diversos pesquisadores (HILLOCKS, 1986; KEH, 1990; LEKI, 1990; COHEN, 1987). Estes pesquisadores não demonstram um consenso em seus estudos. O estudo realizado por Cohen (1987) para verificar como os alunos reagiam frente ao feedback das redações ofertados pelos professores constatou que os comentários não eram claros, não eram úteis para o desenvolvimento do aluno e que, na maioria das vezes, referiam-se à gramática. Hahn (1981) revela que os alunos, com muita frequência, não entendem os comentários recebidos, e os que conseguem decifrar as mensagens, simplesmente não sabem o que fazer com elas. Para que isto não ocorra, e para que o feedback seja eficaz, Cohen (1987) recomenda que os professores ensinem aos alunos sobre como receber 0 feedback, e como ele deve proceder frente aos comentários e instruções.

Entretanto, nota-se que há um consenso de que o feedback é uma ferramenta fundamental no processo de aquisição de língua estrangeira porque, muito frequentemente, é o principal canal de comunicação entre o professor e o aluno. Assim sendo, os professores estarão desperdiçando uma grande chance de intervenção se o feedback for mal conduzido, se não for esclarecedor, se não oferecer um modelo adequado de como se deve articular o idioma, se não acontecer em um contexto apropriado (como no cotidiano de sala de aula, em grupo, em momentos individuais), ou se não houver equilíbrio entre o elogio e a crítica (CRUSE; FONTES, 2011).

Segundo Sherman (1994), o feedback pode ser oferecido através de três formas distintas: correções realizadas pelo professor, comentários feitos pelo mesmo e/ou uma nota ou um conceito. O feedback, de acordo com Gus, (2001), possui dois componentes distintos: a avaliação e a correção. feedback avaliativo é comumente ofertado através de uma nota ou um conceito, seguido ou não de um breve comentário. O feedback corretivo, por sua vez, refere-se a aspectos informativos fornecidos sobre o desempenho do aluno, isto é, as correções realizadas pelo professor orientando o aluno acerca daquilo que estava certo ou errado, indicando 
maneiras de corrigir e descrevendo a natureza do erro (Gus, 2001).

A correção das redações ou de atividades escritas realizadas entre os pares é denominada peer feedback. Thac Sĩ (2008) argumenta que a utilização do peer feedback aumenta o interesse do aluno em escrever, pois seu público deixará de ser irreal (o professor), e alcançará um público real (seus pares). Ainda segundo o autor, há evidência de que a motivação em escrever aumenta o desempenho do aluno e de que a aprendizagem colaborativa exerce um papel formativo durante $o$ processo de aprendizagem.

Derham (1995) afirma que o peer feedback reduz a dependência do professor e o aluno tem a oportunidade de desenvolver o princípio da autonomia. Segundo Charles (1990), o feedback ideal é aquele em que o professor tem a oportunidade de reunir-se individualmente com cada aprendiz, dispõe de um ambiente favorável para dialogar, fazer sugestões e ouvir possíveis questionamentos por parte do aprendiz. É um momento propício para que haja o estreitamento da relação de confiança e de comprometimento entre o professor e o aprendiz. Esta forma de feedback é denominada "conferência". A conferência permite uma personalização do feedback (JAMES, 1998) e torna cada aprendiz único naquele momento.
A verdade é que não há uma única forma ideal de feedback. O importante é que o feedback seja promovido de forma clara e útil para o aluno. Assim sendo, o professor estará exercendo seu papel de mediador do conhecimento e alimentando o aluno com sugestões e caminhos que têm por objetivo fazê-lo refletir sobre o seu desempenho, e, assim, fazê-lo confirmar ou redirecionar as diretrizes de sua própria aprendizagem. Para que isso efetivamente aconteça, deve existir um constante diálogo entre o professor e o aluno (HYLAND, 1990 e SHERMAN, 1994).

\section{A AUTOEFICÁCIA}

A situação de aprendizagem em sala de aula é uma atividade notoriamente complexa em função do ambiente pluridimensional que lhe é inerente. Bzuneck (2000) salienta que a função de ensinar enfrenta obstáculos, reveses, frustrações e fracassos devido a sua natureza complexa. O autor ressalta alguns fatores que levam os alunos a não desenvolverem a eficácia no ensino, tais como: alunos desmotivados; alunos com defasagem educacional; alunos com baixo rendimento; turmas com muitos alunos; baixo envolvimento dos familiares de alunos; falta de apoio dos colegas; estruturas pedagógicas ultrapassadas; falhas do sistema educacional.

Neste contexto, observa-se ainda, o desinteresse do aluno, e consequentemente, 
a sua falta de envolvimento com o seu próprio processo de aprendizagem. Segundo Bzuneck (2001) a desmotivação dos alunos é objeto de queixa de professores de todos os níveis escolares, ou seja, os alunos (ou pelo menos alguns deles) não demonstram ter a dedicação desejável aos estudos, ao contrário, revelam-se indisciplinados. 0 mesmo autor afirma que os professores atribuem um efeito de causalidade pelo fracasso do aluno, isto é, se o aluno não está se desenvolvendo como o esperado é porque está desmotivado.

A falta de motivação tornou-se um problema constante apontado por professores, pela simples constatação de que, alinhado a outros fatores, sua ausência representa pouco investimento pessoal de qualidade nas tarefas educacionais, e ainda gera falta do estabelecimento de metas e de comportamento proativo (GUIMARÃES e BZUNECK, 2008). Nessa perspectiva, torna-se necessário compreender os processos cognitivos inerentes à aprendizagem e, mais especificamente, de que forma as pessoas consideram suas próprias capacidades crenças de autoeficácia - pois são elas que Ihes fornecem condições de enfrentamento nas adversidades.

Com o objetivo de entender a natureza humana, que está em constante busca pelo controle dos acontecimentos que envolvem sua vida, o presente estudo pauta- se na vertente da Teoria Social Cognitiva elaborada por Albert Bandura. Esta teoria preocupa-se em estudar o desenvolvimento humano na perspectiva triádica e bidirecional observada entre o homem, os fatores pessoais e os fatores ambientais (BANDURA 1977, 1986). É alvo dos estudos de Bandura buscar compreender os mecanismos cognitivos que estão presentes nesta interação recíproca. Um dos pontos fundamentais destes processos cognitivos são as crenças de autoeficácia, núcleo central desta pesquisa.

As crenças de autoeficácia estão entre os fatores que constituem os mecanismos psicológicos da motivação do aluno, sendo Bandura $(1977 ; 1986)$ o autor que formulou o conceito, descreveu sua operacionalização e primeiro impulsionou as pesquisas na área. Com dezenas de estudos publicados, Schunk é o autor que individualmente mais se dedicou à aplicação do conceito de autoeficácia no contexto educacional, relacionando suas influências na motivação e na aprendizagem do aluno (PINTRICH e SCHUNK, 1996).

Como o próprio termo sugere, as crenças de autoeficácia fazem parte das expectativas relacionadas ao self. Bandura (1986) define as crenças de autoeficácia como sendo um julgamento que o sujeito faz das suas próprias capacidades em organizar e executar cursos de ação requeridos para se 
atingir certo grau de performance. Segundo Schunk (1991), as crenças de autoeficácia são convicções pessoais de que se é capaz de realizar uma ação ou tarefa em um grau de qualidade específico.

As crenças de autoeficácia constituem em uma avaliação ou julgamento pessoal em relação à própria inteligência, habilidades, conhecimentos etc., que nesta definição de Bandura assumem o termo capacidades. Não se trata de realmente possuir tais capacidades ou não, basta que a pessoa tenha convicção de que as possui. Tais capacidades estão relacionadas à organização e execução das ações, ou seja, o indivíduo revela uma expectativa positiva de conseguir realizar determinada tarefa. Além disso, constata-se um componente de finalidade nas crenças de autoeficácia, pois há que se cumprir determinadas exigências na realização de uma ação específica. Desta forma, as pessoas com robustas crenças de autoeficácia consideram os três componentes acima simultaneamente diante das realizações de suas ações: as próprias potencialidades, o objetivo de cumprir as exigências das tarefas a serem executadas e as ações que levem a esse objetivo.

As pesquisas fundamentadas na Teoria Sociocognitivista de Bandura confirmam a relevância das crenças de autoeficácia para a motivação dos alunos e apontam que o professor exerce função primordial no reconhecimento de sua importância e no seu desenvolvimento (BANDURA, 1977, 1986; BZUNEK, 2001; PAJARES, 1997; SCHUNK, 1989; 1991).

A fim de identificar a real importância das crenças de autoeficácia no contexto educacional, é fundamental compreender como tais crenças se formam ou quais são as suas origens. Mais ainda, Bzuneck (1996) argumenta que, com a intenção de influenciar o comportamento, a condução da aprendizagem e a formação do aluno, o professor deve dedicar-se em compreender o seu sistema de crenças. Bandura (1986) identifica quatro fontes que dão origem a essas crenças: as experiências de êxito, as experiências vicárias, a persuasão verbal e os indicadores fisiológicos. Tais fontes podem estar presentes de forma individual ou combinada. Em decorrência da presença de uma ou mais fontes de crenças de autoeficácia, os alunos podem inferir seu grau de eficácia tanto no início como durante o período de desenvolvimento de qualquer ação, a cada instante ou etapa do trabalho.

As experiências de êxito constituem em uma fonte fundamental na execução de uma tarefa. Bandura (1986) argumenta que uma sucessão de êxitos na realização de ações similares gera ao aluno a informação de que ele é capaz de realizar uma nova tarefa. Por outro lado, fracassos sucessivos Ihe causam uma percepção de falta de 
competência. Quando o aluno infere que tem capacidade de corresponder às expectativas da tarefa que lhe foi proposta, suas crenças de autoeficácia são afirmadas. É importante ressaltar que um fracasso isolado ou eventual seguido de várias experiências bem sucedidas não causará impacto relevante sobre as crenças positivas de autoeficácia. Da mesma forma, uma experiência de sucesso isolada terá pouco impacto no aumento dessas crenças (SCHUNK, 1989).

Nessa perspectiva, pode-se afirmar que uma série de experiências bem sucedidas pode acarretar na promoção das crenças de autoeficácia para aprender. Entretanto, o aluno dificilmente consegue perceber e identificar seu progresso se este acontecer paulatinamente ou se ele não for informado pelo professor através de um feedback positivo e consistente (SCHUNK, 1989).

A persuasão oral constitui outra fonte de crenças de autoeficácia descrita por Bandura (1986). O autor defende que através do recebimento de feedback positivo, principalmente por parte do professor, o aluno poderá desenvolver as crenças de autoeficácia. No entanto, os efeitos dessas crenças serão realmente eficazes se partirem de uma pessoa de credibilidade e, acima de tudo, se o aluno comprovar a veracidade das informações recebidas. Caso contrário, a crença de autoeficácia não se confirmará (BANDURA, 1997).
Assim sendo, o professor deve assumir um papel de relevância na comunicação positiva das reais capacidades dos alunos e evitar situações e verbalizações que venham a gerar dúvidas sobre elas. É imprescindível que o professor ofereça de maneira adequada o feedback de acerca do desempenho e que informe ao aluno com credibilidade, confiança e perícia a respeito de suas potencialidades (BANDURA, 1997).

Considerando que a persuasão oral é uma importante fonte de crença de autoeficácia, Bandura (1995) postula que a sala de aula deve ser um ambiente desprovido de qualquer tipo de comparação social que possa gerar ao aluno a comparação entre os pares. Tal atitude poderá ser prejudicial ao aluno, pois ao se julgar menos capaz, suas crenças de autoeficácia serão afetadas. Portanto, ainda segundo esse autor, a maneira como as aulas forem estruturadas e conduzidas cooperará para o aumento ou a redução das crenças de autoeficácia em cada aluno. Entretanto, é importante ressaltar que as crenças de autoeficácia são consideradas necessárias para a motivação do aluno, porém quando atuam de modo isolado não são suficientes para garantir um desempenho satisfatório nos estudos (SCHUNK, 1991).

Em suma, as crenças de autoeficácia isoladas de outros componentes como os conhecimentos, as habilidades e as 
capacidades pouco podem influenciar no desenvolvimento e na motivação do aluno. Porém, quando o aluno está suprido desses aspectos, as crenças de autoeficácia têm o poder de motivar os alunos porque é em decorrência delas que acontecerão a escolha, o engajamento, a persistência e a direção durante a aprendizagem (BANDURA, 1986).

As pesquisas que investigaram a relevância das crenças de autoeficácia para a motivação do aluno (BANDURA, 1986, 1989 e 1997; BZUNECK, 2001; SCHUNK, 1991; PINTRICH;SCHUNK, 1996; entre outros) correlacionam o uso adequado do feedback por parte do professor e o aumento das crenças de autoeficácia. Ao oferecer um feedback adequado, claro e consistente diretamente nas fontes de origem dessas crenças, o professor estará influenciando o desempenho dos alunos nas aprendizagens, o que resultará em reflexos positivos no resultado final.

\section{CONCLUSÕES}

Conclui-se que não há um só tipo de feedback ideal e que cada atividade proposta requer um tipo diferente de feedback, de acordo com os objetivos dos trabalhos e da crença de autoeficácia do aluno. Isto se deve ao fato de que os alunos reagem de formas diferentes frente à correção, possuem diferentes níveis de motivação pessoal, bem como estilos de aprendizagem divergentes.
Seguindo esta mesma linha de pensamento, ressalta-se o fato de que os próprios professores demonstram preferir um tipo de feedback em detrimento a outro, de acordo com suas experiências vicárias (BANDURA, 1986) e com seu estilo de ensinar (NUNAN; LAMB, 1996).

O sucesso e a eficácia na promoção do feedback está diretamente relacionado à forma como é conduzido. $O$ importante é que o feedback seja promovido com intenção pedagógica clara, que seja útil e esclarecedor e que efetivamente coopere para o desenvolvimento do desempenho do aluno.

\section{REFERÊNCIAS}

ALLWRIGHT, R. L. Problems in the Study of the Language Teacher's Treatment of Learner Errors. In: BURT, M. K.; DULAY, H.C. (Eds.). TESOL'75: New Directions in Second Language Learning, teaching and bilingual education. Washington, DC: DC: TESOL, 1975. p. 96-109.

ANDERSON, J. et al. Cognitive tutors: Lessons Learned. The Journal of the Learning Sciences, v.4, n. 2, p. 167-207. 1995. Disponível em: $<$ http://dx.doi.org/10.1207/s15327809jls040 2 2>. Acesso em: 20 jan. 2015.

BANDURA, A. Self-efficacy: toward a unifying theory of behavioral change. Psychological Review, v. 84, n. 2, 1977. Disponível em: <http://dx.doi.org/10.1037/0033-

295X.84.2.191>. Acesso em: 20 jan. 2015.

BANDURA, A. Social Foundations of Thought \& Action - A Social Cognitive Theory. Englewood Cliffs: Prentice Hall, 1986. 
BANDURA, A. Exercise of personal and collective efficacy in changing societies. In: BANDURA, Albert (ed.) Self-efficacy in Changing Societies. New York, NY: Cambridge University Press, 1995. p.1-45. Disponível em: $<$ http://dx.doi.org/10.1017/CB09780511527 692>.

BANDURA, A. Self-efficacy: the exercise of control. New York: W. H. Freeman and Company, 1997.

BZUNECK, J. A. Crenças de Autoeficácia de Professores do Primeiro Grau e sua Relação com outras Variáveis de Predição e de Contexto. Arquivos Brasileiros de Psicologia, v. 48, n. 4, , p. 57-89, 1996.

BZUNECK, J. A. As crenças de autoeficácia dos professores. In: SISTO, F.F.; OLIVEIRA, G.C.; FINI, L. D. T. (orgs.). Leituras de psicologia para formação de professores. Petrópolis: Velozes, 2000.

BZUNECK, J. A. A motivação do aluno: aspectos introdutórios. In: BORUCHOVITCH, E.; BZUNECK, J. A. A. (Orgs.). A motivação do aluno: contribuições da psicologia contemporânea. Petrópolis: Velozes, 2001.

CARDOSO, A. C. S. Feedback em contextos de ensino-aprendizagem on-line. Feedback in distance education. Linguagens e Diálogos, v. 2, n. 2, p. 17-34, 2011.

CARDOSO, A. C. S. et al.Alto mar. In: PAIVA, V. L. M. de O.; BRAGA, J.; TAVARES, K. C. do A.. (Orgs.). Inglês Instrumental 1. Belo Horizonte: UFMG, 2007, v. 1.

CHARLES, M. Responding to problem in written English using a student selfmonitoring technique. ELT journal, v.44, n. 4, p. 286-293, 1990.

$\mathrm{CHI}, \mathrm{M} . \mathrm{T}$. H., et al. Learning from tutoring. Cognitive Science, v. 25, n. 3 , p. 471-533. 2001. http://dx.doi.org/10.1207/s15516709cog250 $\underline{41}$

COHEN, A. D. Student processing of feedback on their compositions. In: WENDEN, A.; RUBIN, J. (Eds.) Learner strategies in language learning. Englewood Cliffs, NJ: Prentice Hall, 1987. 5, p. 57-69.

COHEN, A. D.; CAVALCANTI, M. C. Feedback on compositions: teacher and student verbal reports. In: KROLL, B. (Ed.). Second language writing: research insights for the classroom. Northridge: Cambrigde University Press, 1990. Cap. 10, p. 155177.http://dx.doi.org/10.1017/cbo97811395 $\underline{24551.015}$

CRISWELL, E.; BYRNES, H.; PFISTER, G. Intelligent automated strategies of teaching foreign language in context. In: M. SWARTZ, M.; YAZDMI, M. (Eds.) Intelligent Tutoring Systems for foreign Language Learning: The bridge to international communication Berlin: Springer-Verlag, 1991. p. 307-319.

CRUSE, R.; FONTES, A. A escrita e o papel do professor. Cenários: Revista de Estudos da Linguagem, v.1, p. 200, 2011.

DHERAM, P. K. Feedback as a two-bullock cart: a case studuing of teaching writing. ELT Journal, v. 49, n.2, p. 160-168, 1995. http://dx.doi.org/10.1093/elt/49.2.160

ELLIS, R. The Study of second language acquisition. Oxford: Oxford University Press,1997.

FLORES, A. M. O feedback como recurso para a motivação e avaliação da aprendizagem na educação a distância. 2009. Disponível em: $<$ http://www.abed.org.br/congresso2009/CD trabalhos/1552009182855.pdf>.Acesso em: 4 abr. 2013.

FERREIRA et al. (2007). A study of feedback strategies in foreign language classrooms. International Journal of Artificial Intelligence 
in Education 17 (2007) 389 -422 389 IOS Press.

GARRETT, N. ICALL and Second Language acquisition. In V. HOLLAND, J. KAPLAN E M. SAMS (Eds.) Intellingent Language Tutors: Theory shaping technology Hillsdale, NJ: Lawrence Erlbaum Associates, 1995. p. 348349.

GUIMARÃES, S. E. R.; BZUNECK, J. A. Propriedades Psicométricas de um Instrumento para Avaliação da Motivação de Universitários. Ciência e Cognição, v.13, 2008.

GUS, C. O feedback avaliativo e corretivo em composições de língua inglesa analisadas por professores não-nativos, Porto Alegre: Dissertação de Mestrado em Estudos da Linguagem - Universidade do Rio Grande do Sul, 2001.

HAHN, J. Student's reaction to teacher's written comments. National Writing Project Network Newsletter, v. 4, n.9, p. 7-10, 1981.

HILLOCKS, G. Research on written composition: new directions for teaching. Urbana, IL: ERIC, 1986.

HUME, G., MICHAEL, J.; ROVICK, A.; e EVENS, $M$. (1996). Hinting as a tactic in one-on one tutoring. The Journal of the Learning Sciences, 5(1), 23-47. http://dx.doi.org/10.1207/s15327809jls0501 $\underline{2}$

HYLAND, K. Providing productive feedback. ELT Journal, v.44, n.4, p. 279-285, 1990. http://dx.doi.org/10.1093/elt/44.4.279

JAMES, Carl. Errors in language learning and use: exploring error analysis. New York: Longman, 1998.

$\mathrm{KEH}, \mathrm{C}$. L. Feedback in the writing process: a model and methods for implementation. ELT
Journal, v.44, n. 4, p. 294-304, 1990. http://dx.doi.org/10.1093/elt/44.4.294

KULHAVY, R. W. Feedback in written instruction. Review of Educational Research, v. 47, n. 1, 1977.http://dx.doi.org/10.3102/0034654304 $\underline{7002211}$

LEKI, I. Coaching from margins: issues in written response. In: KROLL, B. (Ed.). Second language writing: research insights for the classroom. Northridge: Cambridge University Press, 1990. Cap. 4, p. 57-68.

LYSTER, R.; RANTA, L. Corrective feedback and learner uptake: Negotiation of form in Communicative Classrooms. Studies in Second Language Acquisition, v. 19, p. 3766, 1997. http://dx.doi.org/10.1017/s02722631970010 $\underline{34}$

MERRIL, D.; REISER, B.; RANNEY, M. e TRAFTON, J. Effective Tutoring Techniques: Comparison of Human Tutors and Intelligent Tutoring Systems. The Journal of the Learning Sciences, v. 2, n. 3, p. 277-305, 1992.

http://dx.doi.org/10.1207/s15327809j|s0203 $\underline{2}$

NAGATA, N. (1997). The effectiveness of computer-assisted metalinguistic instruction: A case study in Japanese. Foreign Language Annals, 30(2), 187-199.

http://dx.doi.org/10.1111/j.1944-

9720.1997.tb02341.x

NUNAN, D.; LAMB, C. Error correction and feedback. In: NUNAN, D.; LAMB, C. (Eds.). The self-directed teacher: managing the learning process. Cambridge: Cambridge Language Education, 1996. p. 68-79.

PAIVA, V. L. M. O. Feedback em Ambiente Virtual. In: LEFFA, V. (Org.) Interação na aprendizagem das línguas. Pelotas: EDUCAT, 2003. Disponível em: 
<www.veramenezes.com/feedback.htm>.

Acesso em: 28 mar. 2014.

PAJARES, F. Current directions in self-efficacy research. In: MAEHR, M. L.; PINTRICH, P. R. (eds.) Advances in Motivation and Achievement. Greenwich, CT: JAI Press, Inc., v. 10, 1997.

PINTRICH, P. R.; SCHUNK, D. H. Motivation in Education: Theory, Research, and Applications. Englewood Cliffs, N.J.: Prentice Hall, Inc., 1996.

SAMS, M. Advanced Technologies for Language Learning: The Bridge project. In: V. HOLLAND, J. KAPLAN \& M. SAMS (Eds.). Intelligent Language Tutors: Theory shaping technology Hillsdale, NJ: Lawrence Erlbaum Associates, 1995.p. 7-21.

SCHERMAN, J. Feedback. Oxford: Oxford University Press, 1994.

SCHUNK, D. H. Self-efficacy and cognitive skill learning. In: AMES, C.; e AMES, R. (eds.). Research on Motivation Education. Goals and Cognitions. New York: Academic Press, Inc., 1989. v. 3

SCHUNK, D. H. Self-Efficacy and Academic Motivation. Educational Psychologist, v. 26, n. 3/ 4, 1991.

SHUTE, V. Focus on formative feedback. ETS Research e Development. Princeton, NJ, March 2007. Disponível em: $<$ www.ets.org/Media/Research/pdf/RR-0711.pdf> Acesso em: 13 maio 2013.

THẠC SĨ, Luận Văn. The impact of online peer feedback on EFL learner's motivation in writing performance: a case study at Can Tho University, 2008. Disponível em: <http://www.ctu.edu.vn/guidelines/scientific thesis/part1/Examples/LuanVanCaoHocHM HIEN\%20K11DHCT.pdf>. Acesso em: 4 abr. 2014.
Recebido para publicação em 20/08/2015 Revisado em 08/09/2015

Aceito em 15/09/2015 